\title{
LA NOCIÓN DE CUIDADO EN LA FUNDAMENTACIÓN DISCIPLINAR DE LAS ESPECIALIDADES DE ENFERMERÍA
}

Elitonia Morán Montes de Oca Pasante de la Licenciatura en Enfermeria y Obstetricia:

"Asesora Lic Enf. Marqanta Cárdenas Jiménez, Responsable del provecto PAPIIT IN312503

\section{Resumen}

En este articulo se plantea el proceso de fundamentación disciplinar de las especialidades en la ENEO de la UNAM desde la perspectiva teórica, en su noción del cuidado. La especialización en las escuelas en el nivel de postgrado consiste en la profundización en el campo especifico de la práctica de la Enfermeria para brindar un cuidado de calidad así como desarrollar capacidades de liderazgo y consultoria.

PALABRAS CLAVE: Cuidado, cuidados especializados, especialización en Enfermería, formación, formación especializada.

\section{Abstract}

Within this paper, the disciplinary foundation process for the ENEO-UNAM 's specialities is stated from the theoretical perspective and at its care notion. Specialization studies in post-grade level schools consists on deepening the nursing practice specific field in order to deliver quality care, as well as to develop student's leadership and advisory capabilities.

KEY WORDS: Care, specialized caring, nursing specialization, upbringing, specialized upbringing.

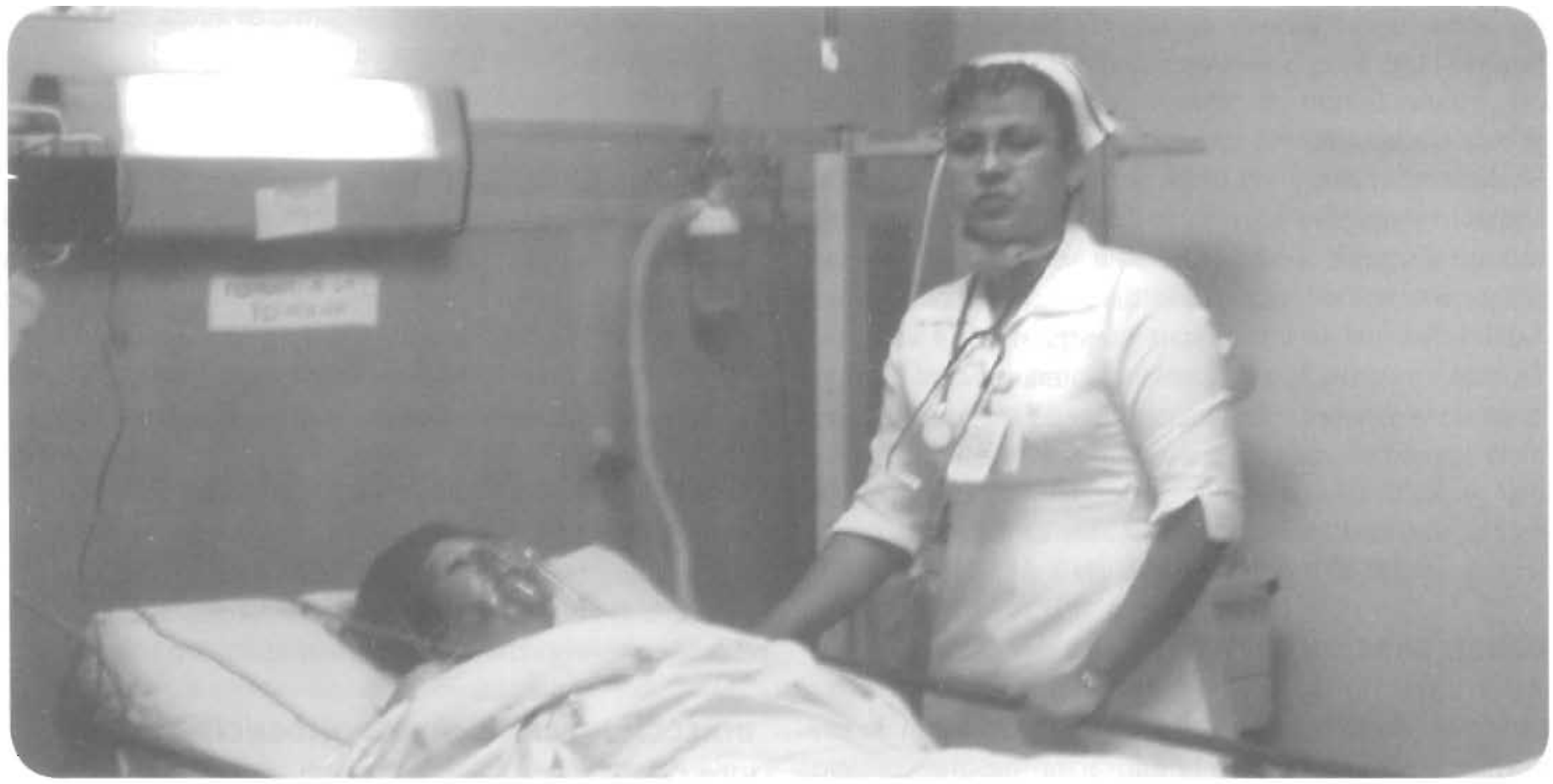




\section{INTRODUCCIÓN}

Una de las opciones de titulación para los egresados de la Licenciatura en Enfermeria es la Actividad de Investigación. El presente trabajo es el resultado de la participación en la Investigación titulada: Evaluación Curricular de las Es. pecialidades en Enfermeria, proyecto PAPIIT IN312503 financiado por la DGAPA.

Dentro de las construcciones teóricas que se han realizado paralelamente a la investigación de campo de corte cualitativa, está la Fundamentación Disciplinar de las Especialidades en Enfermeria, cuyo propósito es que sea un referente más para contextualizar los significados de las entrevistas a profundidad y de las observaciones participantes, asi come para el análisis de la propuesta de aprendizaje establecida en el plan de estudio como documento formal'.

Se parte de la idea que la especialización es un nivel de conocimientos teóricos y prácticos en un área particular de la enfermería, superiores a los adquiridos durante la formación en el pregrado, es asi que una Enfermera especialista es aquella que ha recibido una preparación superior al nivel de Enfermera generalista y en una orientación de la Enfermería especializada; incluye las funciones: clinicas, docentes, administrativas y de consultoria². Está autorizada para ejercer como especialista con conocimientos avanzados en un área especifica de la Enfermería.

Por lo tanto, la Enfermera especiạlista en su intervención directa tiene habilidades para el cuidado especializado, una preparación y experiencia profundas, que le permiten asumir una función independiente, autónoma y ensanchar las fronteras de la práctica de la Enfermeria. Asi, los aspectos distintivos de la práctica especializada de la Enfermeria son la práctica innovadora del cuidado especializado, las capacidades de liderazgo, las habilidades para aplicar los conocimientos científicos a la práctica de la Enfermería así como la capacidad de prestar servicios de consultoria ${ }^{3}$.

Por otra parte, el Consejo internacional de Enfermeria (CIE) señala que los estudios de las especialidades contribuyen a desarrollar los conocimientos y las habilidades de la enfermería. Además la calidad de los cuidados mejora cuando el profesional tiene un centro de interés y este, esta relacionado con la formación de las Enfermeras.

Bajo este contexto, uno de los aspectos fundamentales para el desarrollo de las especializaciones en Enfermería en la ENEO, es la concepción de cuidado que sustenta el proceso de formación, motivo de este trabajo.

\section{NOCIÓN DE CUIDADO}

La noción del cuidado es el eje fundamental en el discurso de la Enfermería especializada, desde el punto de vista disciplinar, el cuidado es el objeto de conocimiento de la Enfermería y criterio fundamental para distinguirla de otras profesiones de la salud, para delimitar su campo de acción en general y en particular del área especializada de que se trate.

El término cuidado* se ha empleado de manera creciente desde la década de los años sesenta hasta nuestros días. Existen diferentes connotaciones en relación al cuidado. Según Colliere", son todạs aquellas acciones que realiza la Enfermera con la intención de que los individuos desarrollen al máximo sus potencialidades con el proposito de mantener, conservar la vida y permitir que esta se continúe considerando sus derechos y sentimientos, de ahi que el cuidado especializado se centre en los valores éticos profesionales no sólo para comprender las alteraciones de las funciones ocasionadas por la enfermedad, sino trascienda hacia el cuidado de la vida de las personas, en los diferentes contextos en que se desarrollan ya que estos son determinantes de su situación de salud.

Siendo el cuidado el centro del saber de Enfermería, cabe señalar que para Colliere este tiene diferentes connotaciones; cuidar de si mismo, cuidar de otros y cuidar con otros $^{5}$

Cuidar de si mismo, implica la responsabilidad que tiene sobre sí mismo la o el profesional de Enfermeria, en el entendido de que no se pude atender las necesidades de los otros sin que se hayan atendido las propias. no se puede ofrecer cuidados profesionales a otros sin que se hayan atendido los personales.Cuidar de otros; es un proceso interactivo por el que la Enfermera y la persona se ayudan mutuamente para desarrollarse, actualizarse y transformarse hacia mayores niveles de bienestar ${ }^{6}$.

Cuidar con otros, es cuando confluyen personas, las personas cuidadas y los cuidadores con costumbres y creencias diferentes las cuales interactúan en el proceso de cuidar hacia un objetivo común, el bienestar.

La acción de cuidar en la práctica especializada no es instrumental; su sentido radica en si misma y en el acto humano que denota; para Paterson y Zderad, Ios elementos del cuidado suponen el encuentro (el ser y la actualización) entre personas (enfermera y persona) en una uransacción subjetiva (estar con y hacer con) que ocurre 
en un tiempo y espacio (tal y como son percibidos por el enfermo y la enfermera) y con una finalidad determinada (bienestar y actualización)?

Las autoras antes citadas consideran que la noción de enfermedad va más allá del desequilibrio biofisiològico, subrayan la relación de ese desequilibrio orgánico con el crecimiento personal y social del individuo; entienden. la enfermedad como un proceso existencial, como una experiencia dinámica y vital de aprendizaje a partir de la cual puede obtenerse un desarrollo personal creciente; esto ocurre cuando la enfermera especialista valora e identifica las capacidades y potencialidades de la persona las cuales hay que desarrollar en el proceso de cuidado. Desde esta visión humanística, el cuidado especializado supone diálogo y construcción de significados compartidos entre la enfermera y la persona pero sobre todo respeto ${ }^{8}$.

Cuidar es una actitud antropológica antes que una técnica; el que la adopta no pretende irrumpir agresivamente en la realidad técnicamente sino dejarla ser; dejar ser al $\operatorname{ser}^{9}$. Ciertamente, cuidar a otro no es sustituirle (aunque a veces sea necesario) sino más bien ayudarle. No consiste en imponerle unas pautas de conductas a la persona si no más bien contribuir a la realización de su proyecto personal, basado en la comprensión.

En sintesis, cuidar, desde la visión de la Enfermería especializada, implica considerar las dimensiones psicológica, emocional, cultural, espiritual y moral de la salud y la en-. fermedad de la persona como una oportunidad única para comprender el significado humano del cuidado a la salud.

\section{OTRA NOCIÓN DE CUIDADO}

Leininger en los 80 s establece los primeros intentos de sistematizar y clarificar conceptualmente la noción de cuidado $^{10}$. Para esta autora existen varias razones para considerar el cuidado como el dominio central del cuerpo de conocimientos y las prácticas de la Enfermería y que justifican la necesidad de realizar investigaciones para su elucidación, afirma que el cuidado se ha manifestado durante. millones de años como fundamental en el crecimiento, desarrollo y supervivencia de los seres humanos.

Caracteriza la misma autora, las diferencias que existen entre los diferentes tipos de cuidado (cuidado genérico; cuidado profesional y cuidados profesional Enfermero), el objetivo de su trabajo es identificar los cuidados presen. tes, implícita o explicitamente, en las creencias, valores y prácticas de los distintos grupos culturales.

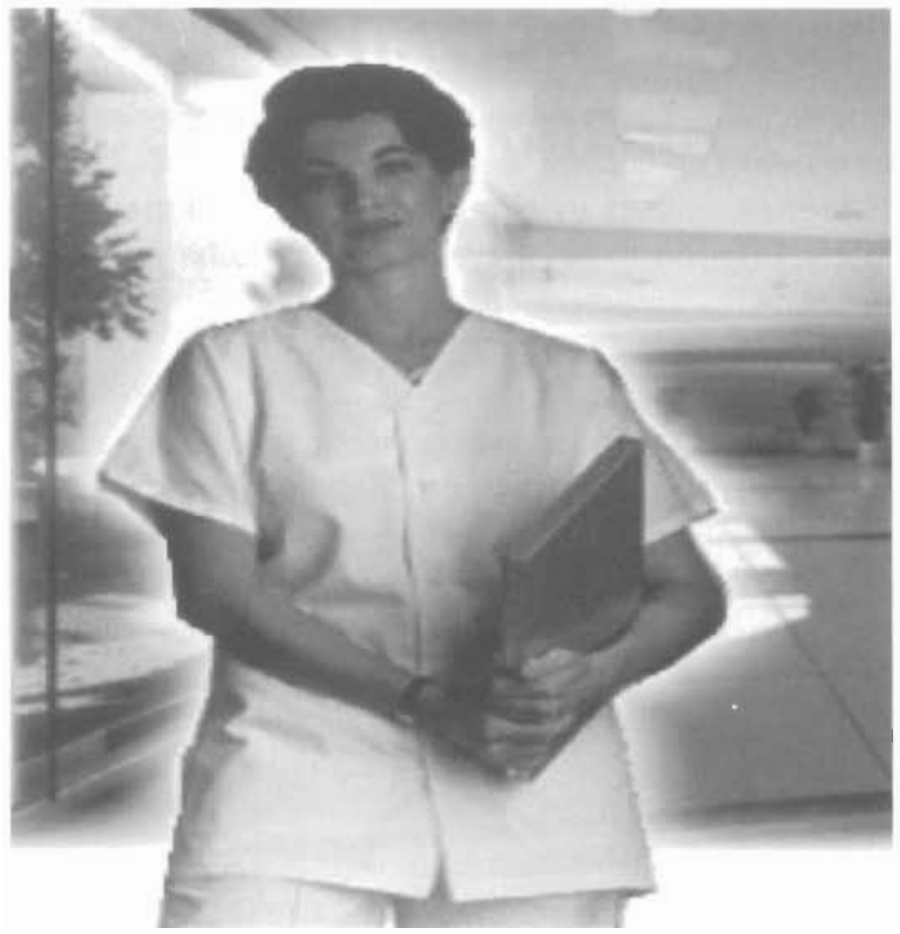

\section{Cuidado genérico:}

Son actos de asistencia, soporte o facilitación prestados a individuos o grupos con necesidades evidentes y orientadas a la mejora y el desarrollo de la condición humana.

La acción de cuidar es de naturaleza universal; en todas las acciones de cuidado el agente manifiesta interés y preocupación por paliar una situación o producir el bienestar en otra persona, llevando a cabo ciertas acciones, un ejemplo de ello es el cuidado y las atenciones que los padres dispensan a los hijos durante la crianza o la preocupación que puede experimentar una persona por un amigo, muchas veces de manera intuitiva.

\section{Cuidados profesionales:}

Son acciones, conductas, técnicas, procesos o patrones aprendidos cognitiva y culturalmente que permiten o ayudan a un individuo, familia o comunidad a mantener o desarrollar condiciones saludables de vida.

Cuando proporciona cuidado profesional el cuidador ejecuta acciones intencionales basadas en un conjunto de saberes (añade a aquellas acciones un componente cientifico) usados para asistir a la persona que recibe el cuidado Ejemplo, los trabajadores sociales y los educadores en general desarrollan acciones de cuidado profesional porque muestran un interés por el desarrollo de las personas y la mejora de su existencia. Sin embargo, no tienen una relación directa con la Enfermería. 
Cuidados profesionales enfermeros:

Son aquellos modos humanisticos y cientificos, aprendidos cognitivamente, de ayudar o educar a individuos, familias o comunidades para recibir servicios personalizados a través de modalidades, culturalmente determinadas, técnicas y proceso de cuidado orientados al mantenimiento y desarrollo de condiciones favorables de vida y de muerte. Esas acciones suponen un interés y una implicación emocional para con el bienestar del otro al que se le reconoce como un ser único.

El cuidado profesional Enfermero tiene la finalidad de mantener y desarrollar la salud y el bienestar de la persona, lo que marca la diferencia con los otros cuidados.

Watson por su parte, considera que es importante citar los fundamentos filosóficos del cuidado partiendo de una perspectiva fenomenológica existencialista y ética para determinar los factores cuidativos que permiten calificar ciertas acciones humanas y afirma que el cuidado está constituldo por acciones transpersonales e intersubjetivas que buscan: proteger, mejorar y preservar la humanidad ayudando a la persona a hallar un significado a la enfermedad, sufrimiento, dolor y existencia y ayudar a otro adquirir autocontrol, autoconocimiento y autocuración"1.

\section{CONCLUSIONES}

- El objeto de estudio de la Enfermería es el cuidado, que se cimienta en la atención que se le brinda a cada persona aplicando, conocimiento teórico - práctico, para ayudar a mejorar el estado de salud de el paciente.

- Existen varias concepciones del cuidado. Todas van encaminadas principalmente al bienestar de la persona y esta se concibe de: forma holistica.

- Las experiencias y conocimientos de la teoría del cuidado, hace que una Enfermera especialista, actúe con habilidades, actitudes y valores profesionales en una practica diferenciada cualitativamente.

- Tener claridad de la propuesta de cuidado en el plan de estudios, es base fundamental para las actividades y la fundamentación de la practica en las especialidades de Enfermería.

- Es importante que las Enfermeras especialistas construyan conocimientos amplios fundamentados sobre la teoría del cuidado, ya que este es la esencia de enfermería e implica la gestión de la calidad de los cuidados que se brindan a las personas, familias y colectivos humanos.

\section{REFERENCIAS BIBLIOGRÁFICAS}

1 Espinosa, Artemisa y Sofla Rodriguez Jiménez, La formación en Enfermeria: pasado y presente. Tendencias Actuales y futuras, Una perspectiva del CIE, Antologia: Docencia en Enfermeria; Ed. SUA, Escuela Nacional de Enfermeria: y Obstetricia UNAM, Méxica, 1998.603 pp.

Ibidem. p. 290

3 bid. p. 301

4

Colliere, F; Marie. Promover la vida. Ed. Interamericana Mc Graw-Hill, México, 1993. 395 Pp.

5 Medina, José Luis, La pedagogia del cuidado. Ed. Alertes, Barcelona, 1999. 307 pp.

6 Ibid. p. 36

$7 \quad 10$

$8 \quad$ bid. p. 38

9 Id

10 Marriner, T; Ann y Martha Baile Alligood, Modelos y Teorías en Enfermeria. Ed. Harcourt Brace, Madrid, 1999. 555 pp.

11 Ibidem. p. 145

\section{NOTA}

* En la literatura anglosajona, la más abundante al respecto, se distingue entre los términos care y caring. El primero hace referencia a la acción de cuidar y el segundo incorpora ạdemás un sentido y una internacionalidad humanitaria en ella.

\section{DIRECCIÓN PARA CORRESPONDENCIA:}

Elitania Morán Montes de Oca: eli_eneo@yahoo.com.mx

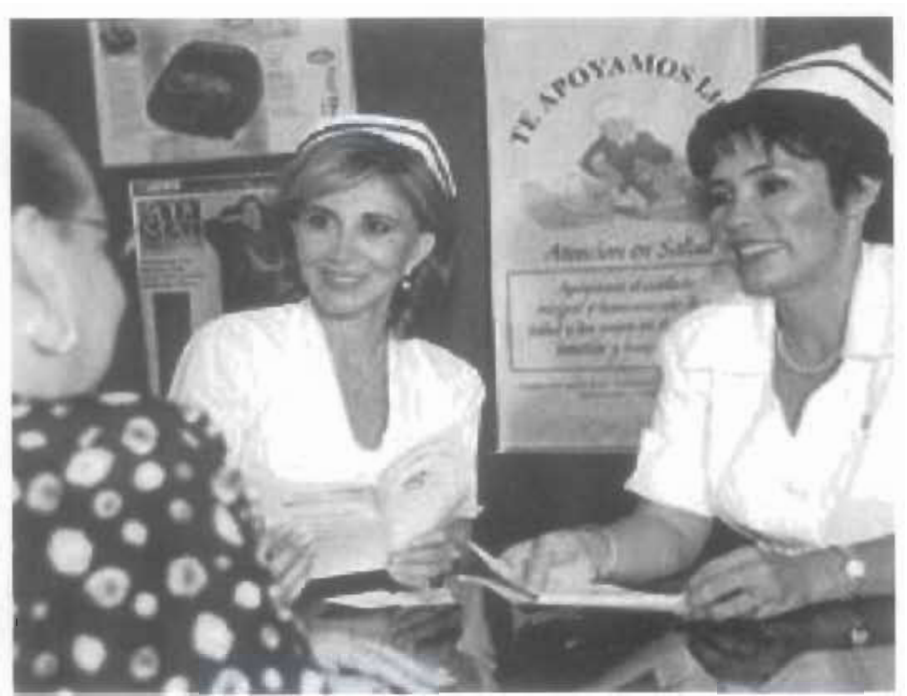

